\title{
XXVI. On the application of legendre's functions to the theory of the Jacobian elliptic integrals
}

\section{J.W. Nicholson B.Sc.}

To cite this article: J.W. Nicholson B.Sc. (1905) XXVI. On the application of legendre's functions to the theory of the Jacobian elliptic integrals, Philosophical Magazine Series 6, 9:50, 264-284, DOI: $10.1080 / 14786440509463278$

To link to this article: http://dx.doi.org/10.1080/14786440509463278

册 Published online: 08 Jun 2010.

Submit your article to this journal

Џ Article views: 5

Q View related articles $๘$ 
264 Mr. Nicholson on Legendre's Functions and the

Copper wire, No. 27 S.W.G. $l=26.50$ cms., mean diameter $.0412 \mathrm{~cm}$. $\therefore$ area $a$ of cross-section $=.00133$ sq. cm., and $\frac{g l^{3}}{a}=1.37 \times 10^{10}$.

\begin{tabular}{|c|c|c|c|c|c|c|c|c|}
\hline $\begin{array}{l}\text { Initial } \\
\text { stretching } \\
\text { force in } \\
\text { dynes. }\end{array}$ & $\begin{array}{l}\text { M. } \\
\text { gr. }\end{array}$ & $\begin{array}{c}y \\
\text { mean. } \\
\text { cms. }\end{array}$ & $\frac{\mathrm{M}}{y}$. & $y^{2}$ & $\frac{\mathbf{M}_{1}}{y_{1}}-\frac{\mathbf{M}}{y}$ & $y_{1}^{2}-y^{2}$ & $\begin{array}{l}\mathbf{M}_{1}-\mathbf{M} \\
y_{1}-y \\
y_{1}{ }^{2}-y^{2}\end{array}$ & $\underset{\epsilon .}{\epsilon y n e s / \mathrm{sq} . c m}$. \\
\hline $650 \mathrm{~g}$ & $\begin{array}{l}15 \\
25 \\
35\end{array}$ & $\begin{array}{l}.2749 \\
\cdot 4024 \\
.5034\end{array}$ & $\begin{array}{l}54 \cdot 56 \\
62.03 \\
69 \cdot 53\end{array}$ & $\begin{array}{l}\cdot 0756 \\
.1619 \\
.2534\end{array}$ & $\begin{array}{r}7 \cdot 47 \\
7 \cdot 50 \\
14.97\end{array}$ & $\begin{array}{l}.0863 \\
.0915 \\
\cdot 1778\end{array}$ & $\begin{array}{l}86 \cdot 6 \\
82 \cdot 0 \\
84 \cdot 2\end{array}$ & $\begin{array}{l}1.19 \times 10^{12} \\
1.12 \\
1.15\end{array}$ \\
\hline $380 \mathrm{~g}$ & $\begin{array}{l}15 \\
25 \\
35\end{array}$ & $\begin{array}{l}\cdot 3630 \\
\cdot 4925 \\
\cdot 5876\end{array}$ & $\begin{array}{l}41 \cdot 32 \\
50 \cdot 76 \\
59 \cdot 55\end{array}$ & $\begin{array}{l}\cdot 1318 \\
.2426 \\
\cdot 3453\end{array}$ & $\begin{array}{r}9 \cdot 44 \\
8 \cdot 80 \\
18 \cdot 24\end{array}$ & $\begin{array}{l}\cdot 1108 \\
\cdot 1027 \\
.213 \overline{3}\end{array}$ & $\begin{array}{l}85 \cdot 2 \\
85 \cdot 7 \\
85 \cdot 4\end{array}$ & $\begin{array}{l}1 \cdot 17 \\
1 \cdot 17 \\
1 \cdot 17\end{array}$ \\
\hline $630 \mathrm{~g}$ & $\begin{array}{l}15 \\
25 \\
35\end{array}$ & $\begin{array}{l}\cdot 2774 \\
\cdot 4044 \\
\cdot 5025\end{array}$ & $\begin{array}{l}54 \cdot 07 \\
61 \cdot 82 \\
69 \cdot 65\end{array}$ & $\begin{array}{l}0769 \\
\cdot 1635 \\
.2525\end{array}$ & $\begin{array}{r}7 \cdot 75 \\
7.83 \\
15.58\end{array}$ & $\begin{array}{l}.0866 \\
.0890 \\
\cdot 1756\end{array}$ & $\begin{array}{l}89 \cdot \overline{0} \\
88 \cdot 0 \\
88 \cdot 7\end{array}$ & $\begin{array}{l}1.23 \\
1.21 \\
1.22\end{array}$ \\
\hline
\end{tabular}

Table of Results.

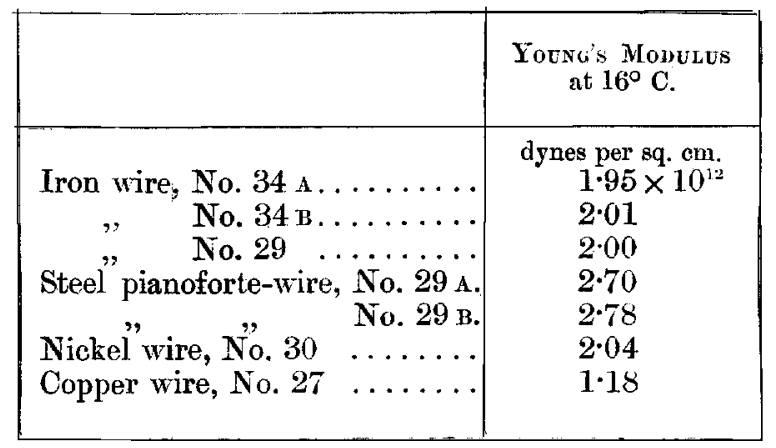

XXVl. Onthe Application of Legendre's Functions to the Theory of the Jacotian Elliptic Integrals. By J. W. NichoLsoN, B.Sc.(Lond. \& Vict.), Trinity College, Cambridge*.

TWHE object of this paper is to indicate a method of some 1 generality, of expanding (1) functions expressible in terms of the complete elliptic integrals of Jacobi ; and (2) definite integrals containing incomplete elliptic integrals in their integrand, in series of Legendre coefficients of increasing

- Communicated by the Author. 
order, and to deduce from the theory certain integrals which are otherwise difficult to evaluate. Most of the results of this latter class, also, are new.

We shall employ the two expressions for $P_{n}(\mu)$, where $\mu=\cos \theta$, which are due to Mehler, viz. :

$$
\begin{aligned}
& \mathrm{P}_{n}(\mu)=\frac{2}{\pi} \int_{0}^{\theta} \frac{\cos \left(n+\frac{1}{2}\right) \phi d \phi}{\sqrt{2(\cos \phi-\cos \theta)}}, \ldots . . \\
& \mathrm{P}_{n}(\mu)=\frac{2}{\pi} \int_{\theta}^{\pi} \frac{\sin \left(n+\frac{1}{2}\right) \phi d \phi}{\sqrt{2(\cos \theta-\cos \phi)}}, . . .
\end{aligned}
$$

and in all future work $\theta$ is considered to be a positive angle, lying between 0 and $\pi$.

Let $F(z)$ be a function of $z$, satisfying the conditions for a Fourier expansion between $z=z_{1}, z=z_{2}$, the expansion having one of the two forms :-

$$
\begin{aligned}
& \mathbf{F}(z)=\sum_{n=0}^{\infty} a_{n} \cos (2 n+1) z, \\
& \mathrm{~F}(z)=\sum_{n=0}^{\infty} a_{n} \sin (2 n+1) z
\end{aligned}
$$

Then between the limits $2 z_{1}, 2 z_{2}, F\left(\frac{\phi}{2}\right)$ has one of the forms

$$
\begin{aligned}
& \mathrm{F}\left(\begin{array}{l}
\phi \\
2
\end{array}\right)=\sum_{0}^{\infty} a_{n} \cos \left(n+\frac{1}{2}\right) \phi, \ldots . \\
& \mathrm{F}\left(\frac{\phi}{2}\right)=\sum_{0}^{\infty} a_{n} \sin \left(n+\frac{1}{2}\right) \phi \ldots . .
\end{aligned}
$$

Multiplying the first form by $\frac{2}{\pi} \cdot \frac{1}{\sqrt{2(\cos \phi-\cos \theta)}}$ and integrating from 0 to $\theta$, supposed not to include $2 z_{1}$, or $2 z_{2}$,

$$
\therefore \quad \frac{2}{\pi} \int_{0}^{\theta} \frac{F\left(\frac{\phi}{2}\right) d \phi}{\sqrt{2(\cos \phi-\cos \theta)}}=\sum_{0}^{\infty} a_{n} \mathrm{P}_{n}(\mu) . .
$$

Similarly, the second form gives

$$
\frac{2}{\pi} \int_{\theta}^{\pi} \frac{\mathrm{F}\left(\frac{\phi}{2}\right) d \phi}{\sqrt{2(\cos \theta-\cos \phi)}}=\sum_{0}^{\infty} a_{n} \mathrm{P}_{n}(\mu) . .
$$

These formulæ will be applied to several Fourier expansions. The latter will be freely quoted, and are all to be found in 
Whittaker, 'Modern Analysis,' Chap. vii., or a similar work.

The simplest case is the series

$$
\cos \frac{\phi}{2}-\cos \frac{3 \phi}{2}+\cos \frac{5 \phi}{2} \ldots \ldots
$$

which we shall not dwell upon.

The expansion produced is

$$
\frac{1}{2 \cos \frac{\theta}{2}}=\mathrm{P}_{0}(\mu)-\mathrm{P}_{1}(\mu)+\mathrm{P}_{2}(\mu) \ldots+(-)^{n} \mathrm{P}_{n}(\mu)+\ldots
$$

where $\theta$ is not zero or $\pi$, but may have any intermediate value. Therefore, by the integral properties of Legendre's functions, the integral on left being finite at both limits,

$$
\frac{1}{2} \int_{-1}^{1} \frac{\mathrm{P}_{n}(\mu) d \mu}{\cos \frac{\theta}{2}}=(-)^{n} \frac{2}{2 \cdot n+1}
$$

or

$$
\int_{0}^{\pi} P_{n}(\cos \theta) \sin \frac{\theta}{2} d \theta=(-)^{n} \frac{2}{2 n+1} . . .
$$

The next case is the result:

$$
\frac{\pi}{4}=\sin z+\frac{1}{3} \sin 3 z+\frac{1}{5} \sin 5 z+\ldots \ldots
$$

holding from $z=0$ to $\pi$, both exclusive.

Therefore between $\phi=0$ and $2 \pi$,

Hence

$$
\frac{\pi}{4}=\sum_{0}^{\infty} \frac{\sin (2 n+1) \frac{\phi}{2}}{2 n+1}
$$

$$
\begin{aligned}
\sum_{0}^{\infty} \frac{P_{n}(\mu)}{2 n+1} & =\frac{2}{\pi} \sum_{0}^{\infty} \frac{1}{2 n+1} \int_{\theta}^{\pi} \frac{\sin (2 n+1) \frac{\phi}{2}}{\sqrt{2(\cos \theta-\cos \phi)}} \\
& =\frac{1}{4} \int_{\theta}^{\pi} \frac{d \phi}{\sqrt{\cos ^{2} \frac{\theta}{2}-\cos ^{2} \frac{\phi}{2}}} ;
\end{aligned}
$$

the excluded values of $\phi$ being outside the range of integration. 


$$
\begin{aligned}
& \text { Put } \tan \frac{\phi}{2}=\tan \frac{\theta}{2} \operatorname{cosec} \lambda \text {, } \\
& \therefore \quad d \phi=-\frac{2 \tan \frac{\theta}{2} \cdot \operatorname{cosec} \lambda \cot \lambda d \lambda}{1+\tan ^{2} \frac{\theta}{2} \operatorname{cosec}^{2} \lambda} \\
& \cos ^{2} \frac{\theta}{2}-\cos ^{2} \frac{\phi}{2}=\frac{\sin ^{2} \frac{\theta}{2} \cot ^{2} \lambda}{1+\tan ^{2} \frac{\theta}{2} \operatorname{cosec}^{2} \lambda} \\
& \therefore \int_{\theta}^{\pi} \frac{d \phi}{\sqrt{\cos ^{2} \frac{\theta}{2}-\cos ^{2} \frac{\phi}{2}}}=2 \int_{0}^{\frac{\pi}{2}} \frac{\sec \frac{\theta}{2} \operatorname{cosec} \lambda d \lambda}{\sqrt{1+\tan ^{2} \frac{\theta}{2} \operatorname{cosec}^{2} \lambda}} \\
& =2 \mathrm{~K}\left(\cos \frac{\theta}{2}\right) \text {, }
\end{aligned}
$$

where $\mathbf{K}(k)$ is Jacobi's complete integral $\int_{0}^{\frac{\pi}{2}} \frac{d \lambda}{\sqrt{1-k^{2} \sin ^{2} \lambda}}$.

\section{Hence}

$$
\mathrm{K}\left(\cos \frac{\theta}{2}\right)=2 \sum_{0}^{\infty} \frac{\mathrm{P}_{n}(\mu)}{2 n+1} . . . .
$$

where $\mu=\cos \theta$.

The formula

$$
\frac{\pi}{4}=\cos z-\frac{1}{3} \cos 3 z+\frac{1}{5} \cos 5 z \ldots \ldots
$$

holds from $z=-\frac{\pi}{2}$ to $z=+\frac{\pi}{2}$, both exclusive.

$$
\therefore \quad \frac{2}{\pi} \int_{0}^{\theta} \frac{\pi}{4} \cdot \frac{d \phi}{\sqrt{2(\cos \phi-\cos \theta)}}=\sum_{0}^{\infty} \frac{(-)^{n}}{2 n+1} \mathrm{P}_{n}(\mu) .
$$

To transform this integral, put $\tan \frac{\phi}{2}=\tan \frac{\theta}{2} \cos \lambda$.

$$
\begin{aligned}
\therefore \quad d \phi & =\frac{-2 \tan \frac{\theta}{2} \cdot \sin \lambda d \lambda}{1+\tan ^{2} \frac{\theta}{2} \cos ^{2} \lambda} \\
\cos ^{2} \frac{\phi}{2}-\cos ^{2} \frac{\theta}{2} & =\frac{\sin ^{2} \frac{\theta}{2} \sin ^{2} \lambda}{1+\tan ^{2} \frac{\theta}{2} \cos ^{2} \lambda}
\end{aligned}
$$


268 Mr. Nicholson on Legendre's Functions and the

$$
\begin{aligned}
\therefore \quad \int_{0}^{\theta} \frac{d \phi}{\sqrt{\cos ^{2} \frac{\phi}{2}-\cos ^{2} \theta}} & =2 \int_{0}^{\frac{\pi}{2}} \frac{d \lambda}{\sqrt{1-\sin ^{2} \frac{\theta}{2} \sin ^{2} \lambda}} \\
& =2 K\left(\sin \frac{\theta}{2}\right) .
\end{aligned}
$$

Hence

$$
\mathrm{K}^{\prime}\left(\cos \frac{\theta}{2}\right)=\mathrm{K}\left(\sin _{\frac{\theta}{2}}^{\theta}\right)=2 \sum_{0}^{\infty} \underset{2 n+1}{(-)^{n}} \mathrm{P}_{n}(\mu) .
$$

It follows from (8) that

$$
\begin{aligned}
\text { or } \quad & \int_{-1}^{1} \mathrm{P}_{n}(\mu) \mathrm{K}\left(\sqrt{\frac{1+\mu}{2}}\right) d \mu=\frac{4}{(2 n+1)^{2}} \ldots \\
& \int_{0}^{1} k \mathrm{KP}_{n}\left(2 k^{2}-1\right) d k=\frac{1}{(2 n+1)^{2}} \ldots \ldots .
\end{aligned}
$$

Now when $n$ is large,

$$
P_{n}(\mu)=\sqrt{\frac{2}{n \pi \sin \theta}} \cdot \cos \left\{\left(n+\frac{1}{2}\right) \theta+\frac{\pi}{4}\right\} .
$$

and the series (8) and (9) are accordingly absolutely convergent between $\theta=0$ and $\pi$, but at these limits the theorems are not true. The integrals further deduced, however, may be taken over the entire range, the integrals being finite at each limit.

Squaring (8) and integrating with respect to $\mu$,

$$
\begin{aligned}
\int_{-1}^{1} \mathrm{~K}^{2}(k) d \mu & =4 \sum_{0}^{\infty} \int_{-1}^{1} \frac{\mathrm{P}_{n}^{2}(\mu) d \mu}{(2 n+1)^{2}}+4 \sum_{0}^{\infty} \int_{-1}^{1} \frac{\mathrm{P}_{n}(\mu) \cdot \mathrm{P}_{m}(\mu) d \mu}{(2 n+1)(2 m+1)} \\
& =8 \sum_{0}^{\infty} \frac{1}{(2 n+1)^{3}}
\end{aligned}
$$

Let

$$
\begin{array}{r}
\sigma_{2 m+1}=\frac{1}{1^{2 m+1}}+\frac{1}{2^{2 m+1}}+\frac{1}{3^{2 m+1}}+\ldots \\
\therefore \quad \int_{0}^{1} k \mathrm{~K}^{2} d k=\frac{7}{4} \sigma_{3} . . .
\end{array}
$$

The formulæe arising in a similar way from (9) are

$$
\begin{gathered}
\int_{-1}^{1} \mathrm{P}_{n}(\mu) \cdot \mathrm{K}^{\prime}\left(\sqrt{\frac{1+\mu}{2}}\right) d \mu=(-)^{n} \frac{4}{(2 n+1)^{2}} . \\
\int_{0}^{1} k K^{\prime} \mathbf{P}_{n}\left(2 k^{2}-1\right) d k=\frac{(-)^{n}}{(2 n+1)^{*}} . . .
\end{gathered}
$$


and

$$
\int_{0}^{1} k \mathrm{~K}^{\prime 2} d k=\frac{7}{4} \sigma_{3} \quad . \quad . \quad . \quad .
$$

and we note that if $n$ is even,

and also that

$$
\int_{0}^{1} k\left(\mathrm{~K}-\mathrm{K}^{\prime}\right) \mathrm{P}_{n}\left(2 k^{2}-1\right) d k=0 \quad . \quad .
$$

$$
\int_{0}^{1} k\left(\mathbf{K}^{2}-\mathbf{K}^{\prime 2}\right) d k=0 \ldots . . .
$$

Combining (7) and (8)

$$
\begin{aligned}
\int_{0}^{\pi} \mathrm{K}\left(\cos \frac{\theta}{2}\right) \sin \frac{\theta}{2} d \theta & =2 \sum_{0}^{\infty} \frac{1}{2 n+1} \int_{0}^{\pi} \mathrm{P}_{n}(\cos \theta) \sin \frac{\theta}{2} d \theta \\
& =4 \sum_{0}^{\infty} \frac{(-)^{n}}{(2 n+1)^{2}} \\
\therefore \quad \int_{0}^{2} \mathrm{~K} d k & =2\left(1-\frac{1}{3^{2}}+\frac{1}{5^{2}}-\ldots\right) .
\end{aligned}
$$

while

$$
\begin{aligned}
& \int_{0}^{1} K^{\prime} d k=\frac{1}{2} \sum_{0}^{\infty} \frac{4}{(2 n+1)^{2}}, \\
& \therefore \quad \int_{0}^{3} K^{\prime} d k=\frac{\pi^{2}}{4} . . . .
\end{aligned}
$$

Again,

$$
\begin{aligned}
\int_{-1}^{1} \mathrm{KK}^{\prime} d \mu & =\sum_{0}^{\infty} \frac{2}{2 n+1} \int_{-1}^{1} \mathrm{P}_{n}(\mu) \mathrm{K}^{\prime}\left(\sqrt{\frac{1+\mu}{2}}\right) d \mu \\
& =8\left\{1-\frac{1}{3^{3}}+\frac{1}{5^{3}} \ldots\right\} \\
& =8\left(\frac{\pi}{2}\right)^{3} \frac{\mathrm{E}_{1}}{2} \text { ! by the use of Euler's numbers } \\
& =\frac{\pi^{3}}{4} ; \\
\therefore \int_{0}^{1} k K^{\prime} d k & =\frac{\pi^{3}}{16} . . . . . . . . . .(22)
\end{aligned}
$$

Expressions may be found for integrals containing higher powers of $K, K^{\prime}$, but cannot be concisely exhibited.

A theorem will be used, which will be stated as follows, and is easy to prove. 
If $m$ is equal to, or less than $n$,

$$
\mathrm{P}_{m}(z) \mathrm{P}_{n}(z)=\sum_{r=0}^{n} \frac{\mathrm{A}_{r} \mathrm{~A}_{m-r} \mathrm{~A}_{n-r}}{\mathrm{~A}_{m+n-r}}\left(\begin{array}{l}
2 n+2 m-4 r+1 \\
2 n+2 m-2 r+1
\end{array}\right) \mathrm{P}_{n+m-2 r} \quad(z)
$$

where

Now

$$
\mathrm{A}_{r}=\frac{2 m !}{2^{m}(m !)^{2}} \cdot \ldots . \quad . \quad .
$$

$$
\int_{-1}^{1} \mathrm{~K}^{2}\left(\cos \frac{\theta}{2}\right) \mathrm{P}_{n}(\mu) d \mu=\sum_{s=0}^{\infty} \frac{2}{2 s+1} \int_{-1}^{1} \mathrm{~K}\left(\cos \frac{\theta}{2}\right) \mathrm{P}_{s}(\mu) \mathrm{P}_{n}(\mu) d \mu .
$$

Write (23) in the form

$$
\mathrm{P}_{m}(z) \mathrm{P}_{n}(z)=\sum_{r=0}^{m}\left(\lambda_{r}\right)_{n}^{m} \underset{(n+m-2 r)}{\mathrm{P}_{(n)}}(z) . .
$$

where $m \leq n$;

$$
\begin{aligned}
& \therefore \int_{-1}^{1} \mathrm{~K}^{2}\left(\cos \frac{\theta}{2}\right) \mathbf{P}_{n}(\mu) d \mu \\
& =\sum_{s=0}^{n} \frac{2}{2 s+1} \int_{-1}^{1} \mathrm{~K}\left(\cos \frac{\theta}{2}\right) \sum_{r=0}^{s}\left(\lambda_{r}\right)_{n n+s-2 r}^{s} \mathrm{P}(\cos \theta) d \mu \\
& +\sum_{\varepsilon=n+1}^{s=\infty} \frac{2}{2 s+1} \int_{-1}^{1} \mathrm{~K}\left(\cos \frac{\theta}{2}\right) \sum_{r=0}^{n}\left(\lambda_{r}\right)_{s}^{n} \mathrm{P}(\cos \theta) d \mu \\
& =\sum_{s=0}^{n} \sum_{r=0}^{s} \frac{2}{2 s+1}\left(\lambda_{r}\right)_{n}^{s} \cdot \frac{2}{2 n+2 s-4 r+1} \\
& +\sum_{s=n+1}^{\infty} \sum_{r=0}^{n} \frac{2}{2 s+1}\left(\lambda_{r}\right)_{s}^{n} \frac{2}{2 n+2 s-4 r+1} \text {. } \\
& \therefore \quad \int_{0}^{1} k \mathrm{~K}^{2} \mathrm{P}_{n}\left(2 k^{2}-1\right) d k=\sum_{s=0}^{n} \sum_{r=0}^{s} \frac{\left(\lambda_{r}\right)_{n}^{s}}{(2 s+1)(2 n+2 s-4 r+1)} \\
& +\sum_{s=n+1}^{\infty} \sum_{r=0}^{n} \frac{\left(\lambda_{r}\right)_{s}^{n}}{(2 s+1)(2 n+2 s-4 r+1)}
\end{aligned}
$$

and

$$
\begin{gathered}
\int_{0}^{1} k \mathrm{~K}^{3} d k=\frac{1}{4} \int_{-1}^{1} \mathrm{~K}^{2}(k) \cdot \sum_{0}^{\infty} \frac{2}{2 n+1} \mathrm{P}_{n}(\mu) d \mu . \\
\therefore \quad \int_{0}^{1} k \mathrm{~K}^{3} d k=2 \sum_{n=0}^{\infty} \sum_{s=0}^{n} \sum_{r=0}^{s} \frac{\left(\lambda_{r}\right)_{n}^{s}}{(2 s+1)(2 n+1)(2 n+2 s-4 r+1)} \\
+2 \sum_{n=0}^{\infty} \sum_{s=n+1}^{\infty} \sum_{r=0}^{n} \frac{\left(\lambda_{r}\right)_{s}^{n}}{(2 s+1)(2 n+1)(2 n+2 s-4 r+1)} \cdot(27)
\end{gathered}
$$

where $\left(\lambda_{r}\right)_{n}^{s}=\left(\lambda_{r}\right)_{s}^{n}$, and is defined in (25). 
Theory of the Jacobian Elliptic Integrals.

This process is general, and we can theoretically find a general expression for $\int_{0}^{1} k \mathrm{~K}^{r} \mathrm{~K}^{\prime /} d k$, which, however, is of
no importance.

Results will now be ohtained involving the complete elliptic integral

$$
\mathrm{E}(k)=\int_{0}^{\frac{\pi}{2}} \sqrt{1-k^{2} \sin ^{2} \phi} d \phi \quad . \quad .
$$

The formulæ connecting $\mathrm{E}$ and $\mathrm{K}$ are

$$
\begin{aligned}
& k \frac{d \mathrm{E}}{d k}=\mathrm{E}-\mathrm{K}, \quad . \quad . \quad . \quad \\
& \frac{\mathrm{E}}{1-k^{2}}=\mathrm{K}+k \frac{d \mathrm{~K}}{d k} \quad \cdots \quad . \quad .
\end{aligned}
$$

Integrating (10) by parts,

$$
\begin{gathered}
\int_{-1}^{1} \mathrm{P}_{n}(\mu) \mathrm{K}(k) d \mu \\
=\left[-\frac{\mathrm{K}(k)}{n(n+1)}\left(1-\mu^{2}\right) \frac{d \mathrm{P}_{n}}{d \mu}\right]_{-1}^{1}+\frac{1}{n(n+1)} \int_{-1}^{1}\left(1-\mu^{2}\right) \frac{d \mathrm{P}_{n}}{d \mu} \cdot \frac{d \mathrm{~K}(k)}{d k} d \mu \\
=\frac{4}{n(n+1)} \int_{0}^{1} \mathrm{P}_{n}^{\prime}\left(2 k^{2}-1\right) \cdot k^{2} k^{\prime 2} \frac{d \mathrm{~K}}{d k} d k \\
=\frac{4}{n(n+1)} \int_{0}^{1} \mathrm{P}_{n}\left(2 k^{2}-1\right)\left\{\mathrm{E}-k^{\prime 2} \mathrm{~K}\right\} k d k \\
\therefore \int_{0}^{1} k\left\{\mathrm{E}-k^{\prime 2} \mathrm{~K}\right\} \mathrm{P}_{n}^{\prime}\left(2 k^{2}-1\right) d k=\frac{n(n+1)}{(2 n+1)^{2}} \cdot \quad \cdot \quad
\end{gathered}
$$

Therefore changing the variables $k, k^{\prime}$,

$$
\int_{0}^{1} k\left\{\mathrm{E}^{\prime}-k^{2} \mathrm{~K}^{\prime}\right\} \mathrm{P}_{n}^{\prime}\left(2 k^{2}-1\right) d k=(-)^{n-1} \frac{n(n+1)}{(2 n+1)^{2}}
$$

An integration of (30) by parts gives

$$
\frac{4 n(n+1)}{(2 n+1)^{2}}=\left[\left(\mathrm{E}-k^{\prime 2} \mathrm{~K}\right) \mathrm{P}_{n}(\mu)\right]_{-1}^{1}-\int_{-1}^{1} \mathrm{P}_{n}(\mu) \frac{d}{4 k d k}\left\{\mathrm{E}-\mathrm{K} k^{\prime 2}\right\} d \mu .
$$

The integrated term is zero, for $1 t_{k^{\prime}=0} k^{\prime 2} \log \frac{4}{k^{\prime}}=0$, and, again,

$$
\frac{d}{k d k}\left\{\mathrm{E}-\mathrm{K} k^{\prime 2}\right\}=\mathrm{K} \text { by (29). }
$$

$$
\therefore \frac{4 n(n+1)}{(2 n+1)^{2}}=\mathrm{E}(1) \mathrm{P}_{n}(1)-\int_{0}^{1} k \mathrm{KP}_{n}\left(2 k^{2}-1\right) d k \text {, }
$$


272 Mr. Nicholson on Legendre's Functions and the giving

$$
\int_{0}^{1} k \mathrm{KP}_{n}\left(2 k^{2}-1\right) d k=\frac{1}{(2 n+1)^{2}}
$$

which is equation (10) again.

We now proceed to treat (15) in the same way as (10).

The formulæ connecting $\mathrm{E}^{\prime}$ and $\mathrm{K}^{\prime}$ are

$$
\begin{aligned}
& \frac{d \mathrm{E}^{\prime}}{d k}=-\frac{k}{k^{\prime 2}}\left(\mathrm{E}^{\prime}-\mathrm{K}^{\prime}\right) \ldots . . \\
& \frac{d \mathrm{~K}^{\prime}}{d k}=-\frac{1}{k k^{\prime 2}}\left\{\mathrm{E}^{\prime}-k^{2} \mathrm{~K}^{\prime}\right\} . .
\end{aligned}
$$

(15) gives

$$
\begin{gathered}
(-)^{n} \frac{4}{(2 n+1)^{2}}=\left[\frac{-\mathrm{K}^{\prime}(k)}{n(n+1)}\left(1-\mu^{2}\right) \frac{d \mathrm{P}_{n}}{d \mu}\right]_{-1}^{1}+\int_{-1}^{1} \frac{\left(1-\mu^{2}\right)}{n(n+1)} \frac{d \mathrm{P}_{n}}{d \mu} \frac{d}{d \mu}\left(\mathrm{K}^{\prime}\right) d \mu . \\
\therefore \quad \int_{0}^{1}\left(1-\mu^{2}\right) \frac{d \mathrm{P}_{n} d \mathrm{~K}^{\prime}}{d \mu d k} d k=(-)^{n} \frac{4 n(n+1)}{(2 n+1)^{2}},
\end{gathered}
$$

or, by $(32 b)$,

$$
\int_{0}^{1} k\left\{k^{2} \mathrm{~K}^{\prime}-\mathrm{E}^{\prime}\right\} \mathrm{P}_{n}^{\prime}\left(2 k^{2}-1\right) d k=(-)^{n} \frac{n(n+1)}{(2 n+1)^{2}},
$$

a repetition of (31) by a different method.

We now consider another expansion in a series of Legendre functions, commencing with the equation

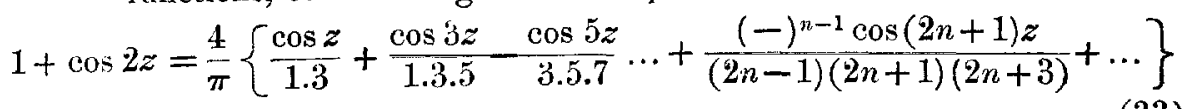

It leads to

$$
\frac{1}{2 \sqrt{2}} \int_{0}^{\theta} \frac{(1+\cos \phi) d \phi}{\sqrt{\cos \phi-\cos \theta}}=\frac{1}{3} P_{0}(\mu)+\sum_{1}^{\infty} \frac{(-)^{n-1} P_{n}(\mu)}{(2 n-1)(2 n+1)(2 n+3)} \text {. }
$$

But

$$
\begin{aligned}
& \frac{1+\cos \phi}{\sqrt{\cos \phi-\cos \theta}}=\sqrt{\cos \phi-\cos \theta}+\frac{1+\cos \theta}{\sqrt{\cos \phi-\cos \theta}}, \\
& \int_{0}^{\theta} \frac{d \phi}{\sqrt{\cos ^{2} \frac{\phi}{2}-\cos ^{2} \frac{\theta}{2}}}=2 K^{\prime}\left(\cos \frac{\theta}{2}\right), \\
& \int_{0}^{\theta} \sqrt{\cos ^{2} \frac{\phi}{2}-\cos ^{2} \frac{\theta}{2}} d \phi=2 \int_{0}^{\frac{\pi}{2} \sin _{\frac{2}{2}} \cdot \frac{\theta}{\left(1+\tan ^{2} \frac{\theta}{2}\right.} \cdot \sin ^{2} \lambda d \lambda}, \\
& \text { th the usual substitution. }
\end{aligned}
$$


Theory of the Jacobian Elliptic Integrals.

If $\cos \frac{\theta}{2}=k$, this becomes

$$
\begin{gathered}
2 \int_{0}^{\frac{\pi}{2}} \frac{k^{2} k^{\prime 2} \sin ^{2} \lambda d \lambda}{\left(1-k^{\prime 2} \sin ^{2} \lambda\right)^{\frac{3}{2}}}=-4 k^{2} k^{\prime 2} \frac{d \mathrm{~K}^{\prime}}{d k^{2}} \\
=2\left(\mathrm{E}^{\prime}-k^{2} \mathrm{~K}^{\prime}\right) \text { by }(32 b) . \\
\therefore \quad \frac{1}{3} \mathrm{P}_{0}(\mu)+\sum_{1}^{\infty} \frac{(-)^{n-1} \mathrm{P}_{n}(\mu)}{(2 n-1)(2 n+1)(2 n+3)} \\
=\frac{1}{2 \sqrt{2}} \cdot\left\{2 \sqrt{2}\left(\mathrm{E}^{\prime}-k^{2} \mathrm{~K}^{\prime}\right)+\frac{(1+\cos \theta)}{\sqrt{2}} \ldots 2 \mathrm{~K}^{\prime}\right\} \\
=\mathrm{E}^{\prime}-k^{2} \mathrm{~K}^{\prime}+\frac{2 k^{2} \cdot \sqrt{2}}{2 \sqrt{2}} \mathrm{~K}^{\prime} \\
=\mathrm{E}^{\prime} . \\
\therefore \quad \mathrm{E}^{\prime}\left(\cos \frac{\theta}{2}\right)=\sum_{0}^{\infty} \frac{(-)^{n-1} \mathrm{P}_{n}(\mu)}{(2 n-1)(2 n+1)(2 n+3)} \text { if } \mu=\cos \theta .
\end{gathered}
$$

The series being absolutely convergent.

It follows that

$$
\begin{gathered}
\int_{-1}^{1} \mathrm{E}^{\prime}(k) \mathrm{P}_{n}(\mu) d \mu=\frac{(-)^{n-1} 2}{(2 n-1)(2 n+1)^{2}(2 n+3)} \cdot . \\
\therefore \quad \int_{0}^{\pi} \mathrm{E}^{\prime}\left(\cos \frac{\theta}{2}\right) \mathrm{P}_{n}(\cos \theta) \sin \theta d \theta=\frac{(-)^{n-1} 2}{(2 n-1)(2 n+3)(2 n+1)}
\end{gathered}
$$

Combining (7) and (34)

$$
\begin{aligned}
\int_{0}^{\pi} \mathrm{E}^{\prime}\left(\cos \frac{\theta}{2}\right) \sin \frac{\theta}{2} d \theta & =-2 \sum_{0}^{\infty} \frac{1}{(2 n-1)(2 n+3)(2 n+1)^{2}} \\
& =2 \sum_{0}^{\infty} \frac{1}{(2 n+1)^{2}} \text { on reduction } \\
& =\frac{\pi^{2}}{4} . \\
\therefore \quad \int_{0}^{1} \mathrm{E}^{\prime}(k) d k & =\frac{\pi^{2}}{8} . \ldots . . . . .
\end{aligned}
$$

Squaring (34) and integrating,

$$
\int_{-1}^{1} \mathrm{E}^{\prime 2}(k) d \mu=2 \sum_{0}^{\infty} \frac{1}{(2 n-1)^{2}(2 n+3)^{2}(2 n+1)^{3}} .
$$

Phil. Mag. S. b. Voi. 9. No. 50. Feb. 1905. 
274 Mr. Nicholson on Legendre's Functions and the

Now

$$
\begin{aligned}
& \left(\frac{1}{2 n-1}-\frac{2}{2 n+1}+\frac{1}{2 n+3}\right)^{2}=\frac{64}{(2 n-1)^{2}(2 n+1)^{2}(2 n+3)^{2}} . \\
& \therefore \quad \int_{0}^{1}\left(\mathrm{E}^{\prime}(k)\right)^{2} k d k \\
& \quad=\frac{1}{128} \sum_{0}^{\infty} \frac{1}{2 n+1}\left(\frac{1}{2 n-1}+\frac{1}{2 n+3}-\frac{2}{2 n+1}\right)^{2} \\
& \quad=\frac{1}{128} \sum_{0}^{\infty}\left[\frac{1}{2 n+1}\left(\frac{1}{(2 n-1)^{2}}+\frac{1}{(2 n+3)^{2}}+\frac{4}{(2 n+1)^{2}}\right)+\frac{3}{2}\right] \\
& \quad=\frac{1}{32} \sum_{0}^{\infty} \frac{1}{(2 n+1)^{3}},
\end{aligned}
$$

and if $\sigma_{3}=\frac{1}{1^{3}}+\frac{1}{2^{3}}+\frac{1}{3^{3}}+\ldots \ldots$

$$
\therefore \int_{0}^{1}\left(\mathbf{E}^{\prime}(k)\right)^{2} k d k=\frac{7}{256} \sigma_{3} . \quad \text {. . . . . . . }
$$

We now integrate (35) by parts,

$$
\begin{array}{r}
\therefore \quad \frac{(-)^{n-12}}{(2 n-1)(2 n+3)(2 n+1)^{2}}=\left[\frac{-\mathrm{E}^{\prime}(k)}{n(n+1)}\left(1-\mu^{2}\right) \frac{d \mathrm{P}_{n}}{d \mu}\right]_{-1}^{1} \\
+\frac{1}{n(n+1)} \int_{-1}^{1}\left(1-\mu^{2}\right) \frac{d \mathrm{P}_{n}}{d \mu} \frac{d \mathrm{E}^{\prime}(k)}{d \mu} d \mu, \\
\therefore \quad \int_{0}^{1}\left(1-\mu^{2}\right) \frac{d \mathrm{P}_{n}}{d \mu} \frac{d \mathrm{E}^{\prime}}{d k} d k=\frac{(-)^{n-1} 2 n(n+1)}{(2 n-1)(2 n+3)(2 n+1)^{2}},
\end{array}
$$

or

$$
\begin{aligned}
\int_{0}^{1} k^{3}\left(\mathrm{E}^{\prime}-\mathrm{K}^{\prime}\right) \mathbf{P}_{n}^{\prime}\left(2 k^{2}-1\right) d k \\
\quad=\frac{(-)^{n}}{2} \cdot \frac{n(n+1)}{(2 n-1)(2 n+3)(2 n+1)^{2}} .
\end{aligned}
$$

$\therefore \quad$ Changing the variable from $k$ to $k^{\prime}$,

$$
\begin{aligned}
\int_{0}^{1} k\left(1-k^{2}\right) & (\mathrm{E}-\mathrm{K}) \mathbf{P}_{n}^{\prime}\left(2 k^{2}-1\right) d k \\
= & \frac{1}{2} \cdot \frac{n(n+1)}{(2 n-1)(2 n+3)(2 n+1)^{2}} .
\end{aligned}
$$

Combining (40) with (30), and (39) with (31) respectively, we find

$$
\begin{aligned}
\int_{0}^{1} k^{3} \mathrm{EP}_{n}^{\prime}\left(2 k^{2}-1\right) d k & =\frac{n(n+1)}{(2 n+1)^{2}}\left\{1-\frac{1}{2(2 n+3)(2 n-1)}\right\} \\
& =\frac{n(n+1)\left(8 n^{2}+8 n-7\right)}{2(2 n+3)(2 n-1)(2 n+1)^{2}},
\end{aligned}
$$


and

$\int_{0}^{1} k k^{\prime 2} \mathrm{E}^{\prime} \mathrm{P}_{n}^{\prime}\left(2 k^{2}-1\right) d k=\frac{(-)^{n-1}}{2} \cdot \frac{n(n+1)}{(2 n+1)^{2}} \cdot \frac{8 n^{2}+8 n-5}{(2 n-1 ;(2 n+3)}$.

Now in $(34)$, write $(\pi-\theta)$ for $\theta$.

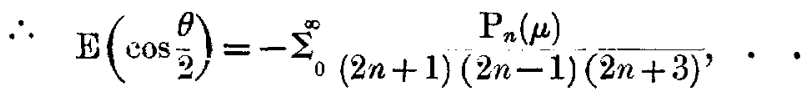

whence

$\int_{0}^{\pi} \mathrm{E}\left(\cos \frac{\theta}{2}\right) \mathrm{P}_{n}(\cos \theta) \sin \theta d \theta=\frac{2}{(2 n-1)(2 n+3)(2 n+1)^{2}}$.

Combining (7) and (43)

$$
\begin{gathered}
\int_{0}^{\pi} \mathrm{E}\left(\cos \frac{\theta}{2}\right) \sin \frac{\theta}{2} d \theta=-2 \Sigma_{0}^{\infty} \frac{(-)^{n}}{(2 n-1)(2 n+3)(2 n+1)^{2}} \\
=\left\{3-\frac{2}{3^{2}}+\frac{2}{5^{2}} \ldots\right\} \\
\therefore \quad \int_{0}^{1} \mathrm{E}(k) d k=\left\{1-\frac{1}{3^{2}}+\frac{1}{5^{2}} \ldots\right\}+\frac{1}{2} \cdot .
\end{gathered}
$$

We note that

while

$$
\begin{aligned}
& \int_{0}^{1} \mathrm{E} d k=\frac{1}{2} \int_{0}^{1} \mathrm{~K} d k+\frac{1}{2}, . . \\
& \int_{0}^{1} \mathrm{E}^{\prime} d k=\frac{1}{2} \int_{0}^{1} \mathrm{~K}^{\prime} d k . . .
\end{aligned}
$$

These might be obtained by integration by parts.

By (43)

$\therefore$ by previous result

$$
\begin{aligned}
\int_{-1}^{1} \mathrm{E}^{2}\left(\cos \frac{\theta}{2}\right) d \mu & =2 \Sigma_{0}^{\infty} \frac{1}{(2 n+1)^{3}(2 n-1)^{2}(2 n+3)^{2}} \\
& =\int_{-1}^{1} \mathrm{E}^{/ 2}\left(\cos \frac{\theta}{2}\right) d \mu,
\end{aligned}
$$

$$
\int_{0}^{2}(\mathrm{E}(k))^{2} k d k=\frac{7}{256} \sigma_{3} . . . .
$$

Again

$$
\int_{-1}^{2} \mathrm{EE}^{\prime} d \mu=\Sigma_{0}^{\infty} \frac{2}{2 n+1} \cdot \frac{(-)^{n}}{(2 n-1)^{2}(2 n+1)^{2}(2 n+3)^{2}}
$$


276 Mr. Nicholson on Legendre's Functions and the

$\therefore \quad \int_{0}^{1} k \mathrm{EE}^{\prime} d k=\frac{1}{2} \sum_{0}^{\infty} \frac{(-)^{n}}{(2 n-1)^{2}(2 n+1)^{3}(2 n+3)^{2}}$

$$
\begin{aligned}
& =\frac{1}{32} \sum_{0}^{\infty} \frac{(-)^{n}}{(2 n+1)^{3}} \\
& =\frac{\pi^{3}}{1024} .
\end{aligned}
$$

Hence

$$
\int_{0}^{1} k \mathrm{EE}^{\prime} d k=\frac{\pi^{3}}{1024}=\frac{1}{64} \int_{0}^{1} k \mathrm{KK}^{\prime} d k . .
$$

Again, by (43) and (9),

$$
\begin{aligned}
\int_{0}^{1} k \mathrm{EK}^{\prime} d k & =-\sum_{0}^{\infty} \frac{1}{(2 n+1)^{2}(2 n+1)(2 n-1)(2 n+3)} \\
& =\Sigma_{0}^{\infty} \frac{(-1)^{n-1}}{(2 n-1)(2 n+3)(2 n+1)^{3}},
\end{aligned}
$$

and

$$
\begin{aligned}
\int_{0}^{1} k \mathbf{E}^{\prime} \mathbf{K} d k & =\Sigma_{0}^{\infty} \frac{(-)^{n-1}}{(2 n-1)(2 n+3)(2 n+1)^{3}} \\
& =\int_{0}^{1} k \mathbf{E K}^{\prime} d k \text {. . . . . . . }
\end{aligned}
$$

Now

$$
\mathrm{EK}^{\prime}+\mathrm{E}^{\prime} \mathrm{K}=\mathrm{KK}^{\prime}+\frac{\pi}{2}
$$

(Cayley, Elliptic Functions, p. 48)

$$
\begin{aligned}
\int_{0}^{1} k \mathrm{EK}^{\prime} d k & =\frac{1}{2} \int_{0}^{3} k\left\{\mathrm{KK}^{\prime}+\frac{\pi}{\overline{2}}\right\} d k \\
& =\frac{1}{2}\left(\frac{\pi^{3}}{16}+\frac{\pi}{4}\right),
\end{aligned}
$$

$\therefore \quad \int_{0}^{1} k \mathrm{EK}^{\prime} d k=\int_{0}^{1} k \mathrm{E}^{\prime} \mathrm{K} d k=\frac{\pi^{3}}{32}+\frac{\pi}{8},$.

Again,

and

$$
\int_{0}^{2} k \mathrm{KE} d k==-\sum_{0}^{\infty} \frac{1}{(2 n-1)(2 n+3) !(2 n+1)^{3}}
$$

$$
\begin{aligned}
& \int_{0}^{1} k \mathrm{~K}^{\prime} \mathrm{E}^{\prime} d k=-\Sigma_{0}^{\infty} \frac{1}{(2 n-1)(2 n+3)(2 n+\mathrm{L})^{3}} ; \\
& \therefore \quad \int_{0}^{1} k \mathrm{KE} d k=\int_{0}^{1} k \mathrm{~K}^{\prime} \mathrm{E}^{\prime} d k \ldots . . .
\end{aligned}
$$


The value of each

$$
\begin{aligned}
& =-\frac{1}{4} \sum_{0}^{\infty}\left(\frac{1}{2 n-1} \frac{1}{2 n+3}\right) \cdot \frac{1}{(2 n+1)^{3}} \\
& =-\frac{1}{4} \sum_{0}^{\infty} \frac{1}{\overline{8}}\left(\frac{1}{2 n-1}+\frac{1}{2 n+3}\right)-\frac{1}{8}\left(\frac{4}{(2 n+1)^{3}}+\frac{2}{(2 n+1)^{2}}+\frac{1}{(2 n+1)}\right) \\
& =\frac{1}{4} \sum_{0}^{\infty} \frac{1}{(2 n+1)^{3}}+\frac{1}{16} \\
& \left.=\frac{7}{32} \sigma_{3}+\frac{1}{16} ; \frac{1}{(2 n+1)^{3}}-\frac{2}{(2 n+1)^{2}}+\frac{1}{2 n+1}\right\} \\
& \quad \therefore \quad \int_{0}^{1} k \mathrm{KE} d k=\int_{0}^{1} k \mathrm{~K}^{\prime} \mathrm{E}^{\prime} d k=\frac{7}{32} \sigma_{3}+\frac{1}{16} . . \quad(53)
\end{aligned}
$$

We may employ the result

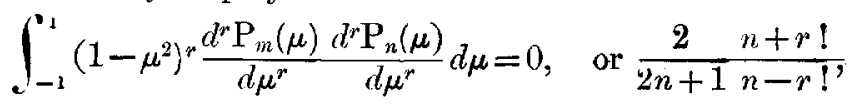

according as $n$ and $m$ are unequal or equal, provided $r$ be a small enough integer. $E$. $g$., we deduce, if $r=1$,

$$
\int_{-1}^{1}\left(1-\mu^{2}\right) \frac{d}{d \mu} \mathbf{K}(k) \cdot \frac{d \mathbf{P}_{n}}{d \mu} d \mu=\frac{2 n(n+1)}{(2 n+1)^{2}},
$$

which coincides with a previous result, and also

$$
\int_{-1}^{1}\left(1-\mu^{2}\right) \frac{d \mathbf{E} d \mathbf{P}_{n}}{d \mu} d \mu=-\frac{2 n(n+1)}{(2 n-1)(2 n+1)^{2}(2 n+3)}
$$

also a previous result.

$$
\text { If } r=2 \text {, }
$$

$$
\begin{gathered}
\int_{-1}^{\rho_{1}}\left(1-\mu^{2}\right)^{2} \frac{d^{2} \mathrm{E} d^{2} \mathrm{P}_{n}}{d \mu^{2}} d \mu=-\frac{2(n-1)(n)(n+1)(n+2)}{(2 n-1)(2 n+1)^{2}(2 n+3)}, \\
\int_{0}^{1} k^{3} k^{\prime 2} \mathrm{E} \cdot \mathrm{P}_{n}^{\prime \prime}\left(2 k^{2}-1\right) d k=-\frac{1}{4} \cdot \frac{(n-1)(n)(n+1)(n+2)}{(2 n-1)(2 n+1)^{2}(2 n+3)}(55)
\end{gathered}
$$

and further particular theorems follow in a similar way, the expansion for $\mathbf{E}^{\prime}$ giving rise to an equal number.

Some applications to incomplete elliptic integrals will now be treated. The elliptic integrals in question occur in the 
278 Mr. Nicholson on Legendre's Functions and the

integ rands of other integrals, and the integration is now with respect to their amplitude, and not their modulus as in the above treatment. $E(\lambda)$ now will denote

$$
\int_{0}^{\lambda} \sqrt{1-k^{2} \sin ^{2} \lambda} d \lambda, \quad \text { and } F(\lambda)=\int_{0}^{\lambda} \frac{d \lambda}{\sqrt{1-k^{2} \sin ^{2} \lambda}} \text {. }
$$
In all cases, the modulus is $k=\cos \frac{\theta}{2}$ for $\mathbf{E}, \mathbf{F}$, and $\sin \frac{\theta}{2}=k^{\prime}$
for $\mathrm{E}^{\prime}, \mathbf{F}^{\prime}$.

If $z$ is between 0 and $\pi$,

But$$
\int_{0}^{0} \frac{\pi d \phi}{\sqrt{\cos ^{2} \frac{\phi}{2}-\cos ^{2} \frac{\theta}{2}}}=2 \pi \mathrm{K}^{\prime}\left(\cos \frac{\theta}{2}\right)
$$$$
=4 \pi \Sigma_{0}^{\infty} \frac{(-)^{n}}{(2 n+1)} P_{n}(\mu) \text { by }(9) ;
$$

Put

$$
\begin{aligned}
& \therefore \quad \int_{0}^{0} \frac{\phi d \phi}{\sqrt{\cos ^{2} \frac{\phi}{2}-\cos ^{2} \frac{\theta}{2}}}=4 \pi \Sigma_{0}^{\infty} \frac{(-)^{n}}{2 n+1} P_{n}(\mu)-8 \Sigma_{0}^{\infty} \frac{P_{n}(\mu)}{(2 n+1)^{2}}(56) \\
& \tan \frac{\phi}{2}=\tan \frac{\theta}{2} \cdot \cos \lambda ;
\end{aligned}
$$

$$
\therefore \quad \int_{0}^{\phi} \frac{d \phi}{\sqrt{\cos ^{2} \frac{\phi}{2}-\cos ^{2} \frac{\theta}{2}}}=2 F^{\prime}(\lambda, k:
$$

$\therefore \quad \int_{0}^{0} \frac{\phi d \phi}{\sqrt{\cos ^{2} \frac{\phi}{2}-\cos ^{2} \frac{\theta}{2}}}=\left[2 \phi F^{\prime}(\lambda)\right]_{0}^{\theta}-2 \int_{0}^{\frac{\pi}{2}} \frac{d \phi}{d \lambda} \cdot F^{\prime}(\lambda) d \lambda$

$$
=4 \int_{0}^{\frac{\pi}{2}} \frac{F^{\prime}(\lambda) \sin \lambda d \lambda}{1+\tan ^{2} \frac{\theta}{2} \cos ^{2} \lambda} \cdot \tan \frac{\theta}{2}
$$

since

$$
\frac{d \phi}{d \lambda}=\frac{-2 \sin \lambda \cdot \tan \frac{\theta}{2}}{1+\tan ^{2} \frac{\theta}{2} \cos ^{2} \lambda}
$$


Theory of the Jacobian Elliptic Integrals.

$\therefore \quad \int_{0}^{\frac{\pi}{2}} \frac{\mathrm{F}^{\prime}(\lambda) \sin \lambda d \lambda}{k^{2}+k^{\prime 2} \cos ^{2} \lambda}=\frac{\pi}{k k^{\prime}} \Sigma_{0}^{\infty} \frac{(-)^{n}}{2 n+1} \mathrm{P}_{n}(\mu)-\frac{2}{k k^{\prime}} \Sigma_{0}^{\infty} \frac{\mathrm{P}_{n}(\mu)}{(2 n+1)^{2}}$,

if $k=\cos \frac{\theta}{2}, \quad \mu=\cos \theta$.

It follows that

$$
\begin{aligned}
\int_{0}^{1} k^{2} k^{\prime} d k \int_{0}^{\frac{\pi}{2}} \frac{F^{\prime}(\lambda) \sin \lambda d \lambda}{k^{2}+k^{\prime 2} \cos ^{2} \lambda} & =\int_{-1}^{1} \frac{\pi-2}{4} d \mu \\
& =\frac{\pi}{2}-1 . . . .
\end{aligned}
$$

Also

$\int_{0}^{1} k^{2} k^{\prime} \mathbf{P}_{n}\left(27 k^{2}-1\right) d k \int^{\frac{\pi}{2}} \frac{\mathbf{F}^{\prime}(\lambda) \sin \lambda d \lambda}{k^{2}+k^{\prime 2} \cos ^{2} \lambda}=\frac{(-)^{n} \pi}{2(2 n+1)^{2}}-\frac{1}{(2 n+1)^{3}}$.

While squaring (57), and calling the integral on the left, $I$,

$$
\begin{aligned}
& \int_{-1}^{1} \mathrm{I} k^{2}{ }^{\prime 2} d \mu=\Sigma_{0}^{\infty}\left(\frac{\pi(-)^{n}}{2 n+1}-\frac{2}{(2 n+1)^{2}}\right)^{2} \frac{2}{2 n+1} \\
& =2 \pi^{2} \cdot \frac{7}{8} \sigma_{3}+\frac{31}{4} \sigma_{5}-8 \pi\left(1-\frac{1}{3^{4}}+\frac{1}{5^{4}} \cdots\right) ; \\
& \int_{0}^{1} \int_{0}^{\frac{\pi}{2}} \int_{0}^{\pi} \frac{k^{3} k^{\prime 2} \mathrm{~F}^{\prime}(\lambda) \mathrm{F}^{\prime}(\mu) \sin \lambda \sin \mu d k d \lambda d \mu}{\left(k^{2}+k^{12} \cos ^{2} \lambda\right)\left(k^{2}+k^{\prime 2} \cos ^{2} \mu\right)} \\
& =\frac{7}{16} \pi^{2} \sigma_{3}+\frac{31}{8} \sigma_{5}-2 \pi\left(1-\frac{1}{3^{4}}+\frac{1}{5^{4}} \cdots\right) \text {. }
\end{aligned}
$$

It may be noted that if

$$
\mathrm{G}(\theta)=\int_{0}^{\theta} \frac{(\pi-\phi) d \phi}{\sqrt{\cos ^{2} \frac{\phi}{2}-\cos ^{2} \frac{\theta}{2}}} ;
$$

then

$$
\begin{aligned}
\int_{0}^{\pi} G^{2}(\theta) \sin \theta d \theta & =64 \Sigma_{0}^{\infty} \frac{2}{(2 n+1)^{5}} \\
& =128\left(1-\frac{1}{2^{5}}\right) \sigma_{5} ; \\
\therefore \quad \int_{0}^{\pi} G^{2}(\theta) \sin \theta d \theta & =124 \sigma_{5} \quad . \quad . \quad . \quad . \quad . .
\end{aligned}
$$

Similar results follow from the series $\cos z-\frac{1}{3^{3}} \cos 3 z+\frac{1}{5^{3}} \cos 5 z \ldots=\frac{\pi}{8}\left(\pi^{2}-z^{2}\right) \quad z$ between $\pm \frac{\pi}{2} ;$ 
280 Mr. Nicholson on Legendre's Functions and the

for

$$
\begin{aligned}
& \begin{aligned}
\Sigma_{0}^{\infty} \frac{(-)^{n}}{(2 n+1)^{3}} P_{n}(\mu) & =\frac{1}{32} \int_{0}^{\theta} \frac{\left(4 \pi^{2}-\phi^{2}\right) d \phi}{\sqrt{\cos ^{2} \frac{\phi}{2}-\cos ^{2} \frac{\theta}{2}}} \\
& =\frac{\pi^{2}}{8} \cdot 2 \mathrm{~K}^{\prime}-\frac{1}{32} \int_{0}^{\theta} \frac{\phi^{2} d \phi}{\sqrt{\cos ^{2} \frac{\phi}{2}-\cos ^{2} \frac{\theta}{2}}}
\end{aligned} \\
& \text { But by previous substitution }
\end{aligned}
$$

$$
\begin{aligned}
& \int_{0}^{\theta} \frac{\phi^{2} d \phi}{\sqrt{\cos ^{2} \frac{\phi}{2}-\cos ^{2} \frac{\theta}{2}}}=\left[2 \phi^{2} F^{\prime}\left(\lambda, \cos \frac{\theta}{2}\right)\right]_{\phi=0}^{\theta} \\
& +16 \int_{0}^{\frac{\pi}{2} F^{\prime}(\lambda) \sin \lambda \tan \frac{\theta}{2} \tan ^{-1}\left(\frac{k^{\prime}}{k} \cos \lambda\right) d \lambda} \frac{1+\tan ^{2} \frac{\theta}{2} \cos ^{2} \lambda}{1}
\end{aligned}
$$

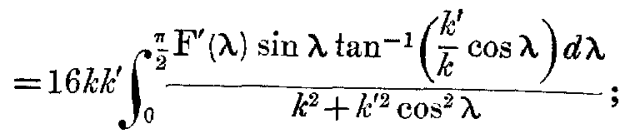

$$
\begin{aligned}
& \therefore \quad \int_{0}^{\pi} \frac{\mathbf{F}^{\prime}(\lambda) \tan ^{-1}\left(\frac{k^{\prime}}{k} \cos \lambda\right) \sin \lambda d \lambda}{k^{2}+k^{\prime 2} \cos ^{2} \lambda}=\frac{\pi^{2}}{k k^{\prime}} \Sigma_{0}^{\infty} \frac{(-)^{n}}{(2 n+1)} \mathrm{P}_{n}(\mu) \\
& +\frac{2}{k k^{\prime}} \mathrm{\Sigma}_{0}^{\infty} \frac{(-)^{n-1}}{(2 n+1)^{3}} \mathrm{P}_{n}(\mu),(62)
\end{aligned}
$$

and if

$$
\begin{aligned}
& G_{1}(\theta)=\int_{0}^{\theta} \frac{\left(4 \pi^{2}-\phi^{2}\right) d \phi}{\sqrt{\cos ^{2} \frac{\phi}{2}-\cos ^{2} \frac{\theta}{2}}} ; \\
& \therefore \quad \int_{0}^{\pi} G_{1}^{2}(\theta) \sin \theta d \theta=(32)^{2} \sum_{0}^{\infty} \frac{2}{(2 n+1)^{7}} \\
&=2 \times(32)^{2} \cdot \sigma_{7}\left(1-\frac{1}{2^{7}}\right) \\
&=2032 \sigma_{7}, . . . . . .
\end{aligned}
$$

and

$$
\begin{array}{r}
\int_{0}^{1} k^{2} k^{\prime} \mathrm{P}_{n}\left(2 k^{2}-1\right) d k \int_{0}^{\frac{\pi}{2}} \frac{\mathrm{F}^{\prime}(\lambda) \sin \lambda \tan ^{-1}\left(\frac{k^{\prime} \cos \lambda}{k}\right) d \lambda}{k^{2}+k^{\prime 2} \cos ^{2} \lambda} \\
=\frac{\pi(-)^{n}}{2(2 n+1)^{2}}+\frac{(-)^{n-1}}{(2 n+1)^{4}}
\end{array}
$$


also

$$
\int_{0}^{1} k^{2} k^{\prime} d k \int_{0}^{\frac{\pi}{2} F^{\prime}(\lambda) \sin \lambda \tan ^{-1}\left(\frac{k^{\prime}}{k} \cos \lambda\right) d \lambda} \frac{\pi}{2}-1 ;(65)
$$

and squaring (62) and integrating to $k$ from 0 to 1 ,

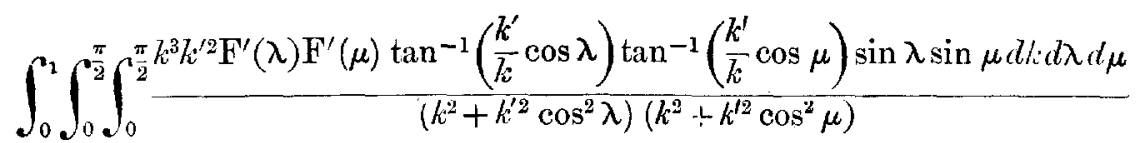

$$
\begin{aligned}
& \left.=\frac{1}{4} \Sigma_{02 n+1}^{\infty} \frac{2}{2 n+1}+\frac{(-)^{2} \pi^{2}}{2 n+1}+\frac{2(-)^{n-1}}{(2 n+1)^{3}}\right)^{2} \\
& =\frac{\pi^{4}}{2}\left(1-\frac{1}{2^{3}}\right) \sigma_{3}+2\left(1-\frac{1}{2^{7}}\right) \sigma_{7}-2 \pi^{2}\left(1-\frac{1}{2^{5}}\right) \sigma_{5} \\
& =\frac{7}{16} \pi^{4} \sigma_{3}+\frac{127}{64} \sigma_{7}-\frac{31}{16} \sigma_{5} . . . . . . . \quad(66)
\end{aligned}
$$

It may be remurked that formulix of these kinds can be successively obtained, and the expansion of the integral

$$
\int_{0}^{\frac{\pi}{2}} F^{\prime}(\lambda)\left[\tan ^{-1}\left(\frac{k^{\prime}}{k} \cos \lambda\right)\right]^{n} \frac{\sin \lambda d \lambda}{k^{2}+k^{12} \cos ^{2} \lambda}
$$

in terms of Legendre's functions, when $n$ is any positive integer, by taking a succession of Fourier series of the kinds already considered in the last two cases.

We may write (56) in a form involving only complete elliptic integrals outside, for

$$
\begin{aligned}
& \int_{0}^{\theta} \frac{\phi d \phi}{\sqrt{\cos ^{2} \frac{\phi}{2}-\cos ^{2} \frac{\theta}{2}}}=\left[\phi \cdot 2 \mathrm{~K}^{\prime} \cos \frac{\phi}{2}\right]_{9}^{\theta}-2 \int_{0}^{\theta} \mathrm{K}^{\prime}\left(\cos \frac{\phi}{2}\right) d \phi \\
\therefore \quad & \int_{0}^{\theta} \frac{\phi d \phi}{\sqrt{\cos ^{2} \frac{\phi}{2}-\cos ^{2} \frac{\theta}{2}}}=4\left\{\mathrm{~K}^{\prime} \cdot \cos ^{-1} k-\frac{1}{2} \int_{0}^{\theta} \mathrm{K}^{\prime}\left(\cos \frac{\phi}{2}\right) d \phi\right\}
\end{aligned}
$$

$\therefore$ by $(56)$,

$$
2 \mathrm{~K}^{\prime} \cos ^{-1} k-\int_{0}^{\theta} \mathrm{K}^{\prime}\left(\cos \frac{\phi}{2}\right) d \phi=2 \pi \Sigma_{0}^{\infty} \frac{(-)^{n}}{2 n+1} \mathrm{P}_{n}(\mu)-4 \Sigma_{0}^{\infty} \frac{\mathrm{P}_{n}(\mu)}{(\boldsymbol{L} n+1)^{2}} ;
$$

$\therefore$ by $(9)$,

$$
\mathrm{K}^{\prime} \cdot\left(2 \pi-\cos ^{-1} k\right)+\frac{1}{2} \int_{0}^{\theta} \mathrm{K}^{\prime}\left(\cos \frac{\phi}{2}\right) d \phi=2 \Sigma_{0}^{\infty} \frac{\mathrm{P}_{n}(\mu)}{(2 n+1)^{2}} .
$$


282 Mr. Nicholson on Legendre's Functions and the

By changing $\theta$ to $\pi-\theta$,

$\mathrm{K}\left(\cos ^{-1} k-\frac{\pi}{2}\right)-\frac{1}{2} \int_{0}^{\pi-\theta} \mathrm{K}^{\prime}\left(\cos \frac{\phi}{2}\right) d \phi=2 \Sigma_{0}^{\infty} \frac{\left(-j^{n}\right.}{(2 n+1)^{2}} \mathrm{P}_{n}(\mu)$.

These with (8) and (9) give the curious results

$$
\begin{array}{r}
\left\{2 \pi-\theta+\frac{1}{2} \int_{0}^{\theta} \frac{\mathrm{K}^{\prime}\left(\cos \frac{\phi}{2}\right)}{\mathrm{K}^{\prime}\left(\cos \frac{\theta}{2}\right)} d \phi\right\} \Sigma_{0}^{\infty} \frac{(-)^{n}}{2 n+1} \mathrm{P}_{n}(\cos \theta) \\
=4 \Sigma_{0}^{\infty} \frac{\mathrm{P}_{n}(\cos \theta)}{(2 n+1)^{2}}, \ldots .
\end{array}
$$

and

$$
\begin{array}{r}
\left\{\theta-\frac{\pi}{2}+\frac{1}{2} \int_{0}^{\pi} \frac{K\left(\cos \frac{\phi}{2}\right)}{K\left(\cos \frac{\theta}{2}\right)} d \phi\right\} \sum_{0}^{\infty} \frac{P_{n}(\cos \theta)}{2 n+1}, \\
=4 \Sigma_{0}^{\infty} \frac{(-)^{n} P_{n}(\cos \theta)}{(2 n+1)^{2}},
\end{array}
$$

which hold from $\theta=0$ to $2 \pi$ except at $\frac{\pi}{2}, \frac{3 \pi}{2}$.

If

$$
\mathrm{I}_{1}=\int_{0}^{\theta} \frac{\mathrm{K}^{\prime}\left(\cos \frac{\phi}{2}\right)}{\mathrm{K}^{\prime}\left(\cos _{2}\right)} d \phi, \quad \mathrm{I}_{2}=\int_{\theta}^{\pi} \frac{\mathrm{K}\left(\cos \frac{\phi}{2}\right)}{\mathrm{K}\left(\cos \frac{\theta}{2}\right)} \cdot d \phi .
$$

We may at once deduce certain integrals as follows:

$$
\begin{aligned}
& \int_{0}^{1} k\left(4 \pi-2 \cos ^{-1} k+\mathrm{I}_{1}\right) \cdot \mathrm{K}^{\prime} \cdot d k=2 . . ., \text {. } \\
& \int_{0}^{2} k\left(I_{2}-\pi+2 \cos ^{-1} k\right) \cdot K \cdot d k=2 \quad . \quad . \quad . \quad \text {. } \\
& \int_{0}^{1} k\left(4 \pi-2 \cos ^{-1} k+\mathrm{T}_{1}\right) \cdot \mathrm{K}^{\prime} \cdot \mathrm{P}_{n}\left(2 k^{2}-1\right) \cdot d k=\frac{7}{4} \sigma_{3} . \\
& \int_{0}^{1} k\left(\mathrm{I}_{2}-\pi+2 \cos ^{-1} k\right) \cdot \mathrm{K} \cdot \mathrm{P}_{n}\left(2 k^{2}-1\right) \cdot d k=\frac{\pi^{3}}{16} . \\
& \int_{0}^{2} k\left(4 \pi-2 \cos ^{-1} k+\mathrm{I}_{i}\right)^{2} \mathrm{~K}^{\prime 2} d k=\frac{31}{4} \sigma_{\bar{j}} \quad \ldots . . \\
& \int_{0}^{1} k\left(\mathrm{I}_{2}-\pi+2 \cos ^{-1} k\right)^{2} \cdot \mathrm{K}^{2} \cdot d k=\frac{31}{4} \sigma_{5} . \quad . \quad . \quad \text {. }
\end{aligned}
$$


From (67) and (7),

$$
\int_{0}^{1}\left(4 \pi-2 \cos ^{-1} k+\mathrm{I}_{1}\right) \cdot \mathrm{K}^{\prime} \cdot d k=4\left(1-\frac{1}{3^{3}}+\frac{1}{5^{3}} \ldots\right)
$$

$\therefore$ by $(21)$,

$$
=\frac{\pi^{3}}{8}
$$

$$
\int_{0}^{1} \mathrm{~K}^{\prime} \cdot\left(\mathrm{I}_{1}-2 \cos ^{-1} k\right) \cdot d k=\frac{7}{8} \pi^{3} . . . .
$$

By (68) and (7),

$$
\int_{0}^{1}\left(2 \cos ^{-1} k-\pi+\mathrm{I}_{2}\right) \cdot \mathrm{K} \cdot d k=\frac{7}{2} \sigma_{3} . \quad . \quad .
$$

Integrating all the above by parts, a large number of integrals involving $\mathrm{E}$ and $\mathrm{K}, \mathrm{E}^{\prime}$ and $\mathrm{K}^{\prime}$ may be deduced.

By combinations of (67) and (68) with previous series of Legendre functions, we may deduce a large number of definite integrals of functions of $k$ from 0 to 1 . The following are examples :

(1) $k \overline{\mathrm{PQ}}\left\{\cos ^{-1} k+\frac{1}{2}\left(\mathrm{I}_{1}, \mathrm{I}_{2}\right)\right\}$ where $\mathrm{P}=\mathrm{K}, \mathrm{K}^{\prime}, \mathrm{E}, \mathrm{E}^{\prime}$

$$
\text { (2) } k \mathrm{PQ}\left\{\sin ^{-1} k+\frac{1}{2}\left(\mathrm{I}_{1}, \mathrm{I}_{2}\right)\right\} \quad, \quad \mathrm{Q}=\mathrm{K}, \mathrm{K}, \mathrm{E}, \mathrm{E}^{\prime}
$$

and these integrals, when found, will on integration by parts give rise to great numbers of elliptic integrals, integrated with respect to their modulus.

It may here be noted, in connexion with the result (62) and similar results, that by a similar investigation with series of the type

$$
\frac{\pi y}{4}=\sin y-\frac{1}{3^{2}} \sin 3 y+\frac{1}{5^{2}} \sin 5 y \ldots
$$

when $y$ is between $\pm \frac{\pi}{2}$, we may in all cases expand, in a series of Legendre functions, the integral

$$
\int_{0}^{\frac{\pi}{2}} \frac{\mathrm{F}(\lambda)\left(\tan ^{-1}\left(\frac{k^{\prime}}{k} \sin \lambda\right)\right)^{n} \cos \lambda d \lambda}{k^{\prime 2}+k^{2} \sin ^{2} \lambda}
$$


where $n$ is a positive integer, and $F(\lambda)$ is the incomplete elliptic integral

$$
\int_{0}^{\lambda} \frac{d \lambda}{\sqrt{1-k^{2} \sin ^{2} \lambda}}
$$

Considering now the equation (62), which may be written

$$
\int_{0}^{\theta} \frac{\phi^{2} d \phi}{\sqrt{\cos ^{2} \frac{\phi}{2}-\cos ^{2} \frac{\theta}{2}}}=8 \pi^{2} \mathrm{~K}^{\prime}-32 \Sigma_{0}^{\infty} \frac{(-)^{n}}{(2 n+1)^{3}} \mathrm{P}_{n}(\mu),
$$

we have

$$
\int_{0}^{\theta} \frac{\phi^{2} d \phi}{\sqrt{\cos ^{2} \frac{\phi}{2}-\cos ^{2} \frac{\theta}{2}}}=\left[2 \phi^{2} K^{\prime}\left(\cos \frac{\phi}{2}\right)\right]_{0}^{\theta}-4 \int_{0}^{\theta} \phi K^{\prime}\left(\cos \frac{\phi}{2}\right) d \phi \text {. }
$$

In this and above, $(66)-(70), \mathrm{K}^{\prime}\left(\cos \frac{\phi}{2}\right)$ denotes an incomplete integral equal to $\mathrm{K}^{\prime}$ when $\phi=\theta$.

$$
\therefore\left(\pi-\cos ^{-1} k\right)\left(\pi+\cos ^{-1} k\right) K^{\prime}+\int_{0}^{\theta} \phi K^{\prime}\left(\cos \frac{\phi}{2}\right) d \phi=4 \Sigma_{0}^{\infty} \frac{(-)^{n}}{(2 n+1)^{3}}\left(\mathrm{P}_{n} \mu\right),
$$

and putting $\pi-\theta$ for $\theta$ in the above,

$$
\begin{aligned}
& \therefore \quad\left[2 \phi^{2} K^{\prime}\left(\cos \frac{\phi}{2}\right)\right]_{0}^{\pi-\theta}-4 \int_{0}^{\pi-\theta} \phi K^{\prime}\left(\cos \frac{\phi}{2}\right) d \phi=8 \pi^{2} K-32 \Sigma_{0}^{\infty} \frac{P_{n}(\mu)}{(2 n+1)^{3}} \\
& \therefore \quad \sin ^{-1} k\left\{\pi+\sin ^{-1} k\right\} \mathrm{K}+\int_{0}^{\pi-\theta} \phi \mathrm{K}^{\prime}\left(\cos \frac{\phi}{2}\right) d \phi=4 \Sigma_{0}^{\infty} \frac{\mathrm{P}_{n}(\mu)}{(2 n+1)^{3}} \cdot . .
\end{aligned}
$$

Proceeding in this manner, it follows that

$$
\sum_{0}^{\infty} \frac{P_{n}(\mu)}{(2 n+1)^{r}}, \quad \Sigma_{0}^{\infty} \frac{(-)^{n} P_{n}(\mu)}{(2 n+1)^{r}}
$$

where $r$ is a positive integer, can be expressed in a similar manner to the above in terms of Jacobian elliptic functions, and their integrals with respect to a function of their amplitude.

The sum of all such series can therefore be concisely expressed. 